\title{
Marocains et Sénégalais de France : permanences et évolution des relations transnationales
}

Moroccans and Senegalese of France: Permanence and Evolution of transnational Relations

Marroquíes y Senegaleses de Francia : permanencias y evolución de las relaciones transnacionales

Thomas Lacroix, Leyla Sall et Monika Salzbrunn

\section{(Q) OpenEdition}

\section{Journals}

Édition électronique

URL : https://journals.openedition.org/remi/4472

DOI : $10.4000 /$ remi.4472

ISSN : $1777-5418$

Éditeur

Université de Poitiers

Édition imprimée

Date de publication : 1 novembre 2008

Pagination : 23-43

ISBN : 978-2-911627-49-0

ISSN : 0765-0752

\section{Référence électronique}

Thomas Lacroix, Leyla Sall et Monika Salzbrunn, « Marocains et Sénégalais de France : permanences et évolution des relations transnationales», Revue européenne des migrations internationales [En ligne], vol. 24 - n² | 2008, mis en ligne le 01 novembre 2011, consulté le 14 avril 2022. URL : http:// journals.openedition.org/remi/4472; DOI : https://doi.org/10.4000/remi.4472 


\title{
Marocains et Sénégalais de France : permanences et évolution des relations transnationales
}

\author{
Thomas LACROIX*, Leyla SALL** \\ et Monika SALZBRUNN****
}

Le maintien des relations des immigrés avec leur pays natal se construit et se structure selon des formes d'échanges diversifiées (visites, contacts téléphoniques, cadeaux... etc.). Ce sont ces échanges qui fondent et forment, à travers la dispersion spatiale, les réseaux transnationaux. Les rapports sociaux transnationaux se matérialisent par des actes concrets dans le quotidien (Salzbrunn, 2002). L'analyse des pratiques est notre clé d'entrée pour comprendre les espaces sociaux transnationaux, l'acteur migratoire dans son environnement (Kaufmann, 2004). Les relations transnationales participent à une mécanique sociale migratoire qui englobe les stratégies d'adaptation psychologiques, spirituelles, culturelles, politiques ou économiques. Il existe donc une interaction très forte entre pratiques et relations sociales qui se déploient par delà les frontières. L'objectif de cet article est d'analyser cette articulation à travers les exemples des Marocains et des Sénégalais résidant en France.

Notre démarche s'inscrit dans la logique des études sur le transnational. Le transnationalisme est couramment défini comme le processus par lequel les migrants construisent un champ social transfrontalier. Alors que les premiers travaux sur les espaces sociaux transnationaux (Glick Schiller, Basch et Szanton Blanc, 1992 ; Glick Schiller et Fouron, 2001 ; Pries, 1996 ; Faist, 2000) mettaient l'accent sur les processus

* Thomas lacroix est chargé de recherche, International Migration Institute, Université d'Oxford, Email : thomas.lacroix@qeh.ox.ac.uk

** Leyla Sall est chercheur associé au Laboratoire MIGRINTER, Migrations Internationales, Espaces et Sociétés, UMR 6588, Email : leylasall@yahoo.fr

*** Monika Salzbrunn est chargée de recherche, CRIA (UMR 8131 CNRS-EHESS), 54 bd Raspail, 75006 Paris, Email : monika.salzbrunn@ehess.fr 
globaux de mise en relation, des orientations plus récentes reviennent vers un enracinement du réseau dans une localité (Glick Schiller, Çağlar, 2006) et dans les pratiques quotidiennes : on parle ainsi d'espace translocal. L'étude comparative des réseaux politiques sénégalais en France et aux États-Unis que Monika Salzbrunn a mené (Salzbrunn 1996, 2002, 2007) montre que l'espace transnational se localise : l'espace urbain est investi de manière durable, puis transformé et habité. Cette approche diachronique et synchronique répond aux critiques formulées par Roger Waldinger (2006 : 26) sur la « dé-historicisation » de ces phénomènes. Notre démarche tente de comprendre les modes d'organisation des collectifs en co-présence et la manière dont les individus se saisissent des occasions liées aux rencontres au sein de l'espace social transnational (Berthomière et Hily, 2006 : 69).

Sur la base de cette approche, nous tenterons d'identifier les pratiques anciennes à travers lesquelles ces champs se sont construits et les activités nouvelles qui dessinent le transnationalisme de demain ${ }^{1}$. Le regard croisé sur les pratiques sénégalaises et marocaines permet de dégager les spécificités de chaque groupe saisi dans le même pays d'accueil, mais il permet surtout d'identifier les grandes constantes observables d'un groupe à l'autre. Nous nous interrogerons plus spécifiquement sur les effets de l'intégration, de l'évolution des flux migratoires et de l'émergence de la seconde génération sur ces pratiques. L'objectif de notre article est d'illustrer l'importance des systèmes communautaires comme socle commun permettant l'instauration et l'évolution des rapports entretenus avec le Sénégal et le Maroc. Nous questionnons finalement les rôles sociaux attribués aux «immigrés », qu'ils soient considérés comme « étrangers » en France ou compatriotes « de l'Éxtérieur » au Maroc et au Sénégal. La dimension conflictuelle des relations avec le groupe d'appartenance (les formes de contrôle social, les différentes générations de l'émigration et de l'immigration) perceptibles dans diverses dimensions des pratiques évoquées dans l'article sera mise en relief. Enfin, nous montrons dans quelle mesure les acteurs participant aux différentes dimensions économiques, politiques et culturelles des pratiques étudiées sont « les mêmes».

Dans les sections qui suivent, après un bref aperçu des migrations sénégalaises et marocaines en France, nous mettrons en évidence la diversité des pratiques politiques, économiques et culturelles des migrants marocains et sénégalais en France. Ce découpage peut paraître artificiel, mais si chacun de ces domaines possède ses propres logiques, tous impliquent très souvent les mêmes acteurs, aux rôles sociaux multiples. La distinction des trois domaines nous permet toutefois de conserver une perspective comparative entre les deux populations. Ceci ouvre la possibilité de dégager les invariants des pratiques transnationales, mais aussi d'évaluer la façon dont les structures sociales affectent de façon différenciée ces pratiques.

1 La recherche a été conduite dans le cadre d'un programme de recherche européen sur les «pratiques transnationales». 


\section{BREF HISTORIQUE DES MIGRATIONS MAROCAINES ET SÉNÉGALAISES EN FRANCE}

L'immigration marocaine est la plus ancienne : elle a un siècle d'histoire (Ray, 1938 ; Belbah and Veglia, 2003). Jusque dans les années 1950, elle est essentiellement organisée par les autorités et le patronat français : d'abord par le gouvernement luimême, pendant la Première Guerre mondiale, afin de combler les besoins de maind'œuvre et de soldats d'un pays en guerre, puis, entre les deux conflits mondiaux, par la Société Générale d'Immigration, institution patronale chargée de répondre aux besoins des entreprises. Les recrutements se font presque exclusivement dans les montagnes berbérophones du Sud marocain. L'immigration marocaine se transforme progressivement au cours des Trente Glorieuses. Les besoins de plus en plus importants de l'industrie française encouragent le développement d'une immigration autoproduite, en marge des canaux officiels. Les zones d'installation se diversifient sur l'ensemble des bassins industriels français. Cette période de l'après-guerre est également celle de l'apparition de nouvelles destinations internationales, notamment la Belgique, les Pays-Bas et la République Fédérale d'Allemagne. À cette expansion européenne fait écho une diversification des régions de provenance. Le Rif, massif montagneux du Nord, supplante le Sud comme principale zone de départ. Au début des années 1960, la signature d'accords entre le Maroc et les principaux pays de destination (en 1963 pour la France) amorce une décennie de départs massifs. La population marocaine atteint 260000 personnes en 1975 (Fondation Hassan II, 2003 : 217). La suspension de l'immigration dite de travail en 1974 ne marque pas la fin de cette expansion. Le regroupement familial et l'accroissement naturel maintiennent les niveaux de croissance : 640852 en 1990 et 728333 en 1999 (chiffre incluant les personnes nées en France de parents marocains) (ibid., 220). Néanmoins, cette constance quantitative recèle une transformation majeure du schéma migratoire marocain. La migration de travail temporaire d'hommes jeunes et illettrés devient, à cette période, une immigration familiale d'installation durable. Ce phénomène d'installation et de diffusion spatiale est le préalable d'une construction diasporique des Marocains de l'étranger. Les relations avec le pays d'origine et les autres pays de destination se formalisent et se diversifient.

La décennie 1990 est marquée par le renforcement de deux tendances : la diversification des pays de destination ${ }^{2}$ et l'extension du comportement migratoire à toutes les couches sociales de la population marocaine. La migration touche désormais l'ensemble des régions, villes comme campagnes, avec une prédominance en faveur du centre du pays (Tadla-Azilal).

La migration sénégalaise présente de nombreuses similitudes. Au cours de cette première période l'émigration est encadrée par l'administration coloniale, et notamment celle des soldats pendant les deux guerres mondiales. Ces premiers

2 L'Espagne et l'Italie ont accueilli environ 500000 personnes sur la décennie (Fondation Hassan II, 2003). Le Canada et les États-Unis émergent également comme nouveaux territoires d'installation. 
arrivants constituèrent les têtes de pont d'une immigration ouvrière et commerçante pendant les Trente Glorieuses dont l'apogée fut atteinte au début des années 1960 avec les migrations ouvrières (soninké et haal poulaar) (Bertoncello et Bredeloup, 1999). Le phénomène migratoire s'est généralisé à l'ensemble du Sénégal dans les années 1980, suite à la crise économique qui frappait le pays. Il s'en est suivi un mouvement de transnationalisation au cours des années 1990. Les lieux de destination se sont diversifiés : l'Espagne, l'Italie et le Portugal depuis le milieu des années 1980 et plus récemment l'Amérique de Nord et l'Australie.

Dans un premier temps, les régions de départ de départ étaient les villes côtières de Dakar, Saint Louis et Rufisque, zones où l'administration coloniale était implantée. Le phénomène est aujourd'hui étendu à l'ensemble du pays. L'immigration commerçante est dominée par la confrérie musulmane des Mourides ${ }^{3}$ mais touche dans une moindre mesure la confrérie Tijâniyya, tandis que les migrations ouvrières sont majoritairement Haal poulaar et Soninkés, originaires de la vallée du fleuve Sénégal.

Selon le recensement général de la population et de l'habitat de 1999, 58815 personnes originaires du Sénégal résidaient alors en France, un chiffre en deçà de la réalité puisque, seuls ceux qui sont en situation régulière sont comptabilisés. Parmi ceux qui sont recensés 19801 sont Français par acquisition et 39014 sont étrangers.

Ce panorama historique permet d'identifier dans le temps les étapes de la transnationalisation de ces deux groupes migratoires. Les années 1990 sont une période de déploiement spatial et d'une forte diversification des pratiques et des modes de circulation. Cependant, les signes de transnationalisation sont perceptibles dès la grande vague migratoire des Trente Glorieuses. Par exemple, l'immigration commerçante Mourides s'amorce dès les années 1960. De fait, les pratiques transnationales sénégalaises (il en va de même pour les Marocains) se sont déployées à partir d'une immigration communautaire ou familiale. Un exemple concerne des jeunes issus de deux villages, Ceedo et Thiaaren. Leurs pères et oncles avaient commencé à explorer et à exercer la vente sur les lieux touristiques que les jeunes investissent actuellement. Leurs parents leur avaient, non seulement, enseigné les rudiments de l'activité commerciale avant de rentrer mais ils leur ont aussi cédé des chambres dans le foyer de la Chevaleret du $13^{\mathrm{e}}$ arrondissement. Le témoignage de l'un des jeunes qui a tenté de s'installer en Grande-Bretagne démontre que sortir de la filière migratoire tissée par la famille suscite aussitôt un rappel à l'ordre de cette dernière.

"Au début je ne voulais pas exercer la vente et mon grand frère qui est en Italie m'a demandé de venir travailler là-bas à Bergame. J'ai travaillé dans une usine de transformation de tomate jusqu'en 2002, puis je suis allé à Londres en

3 La plus visible des quatre confréries soufies présentes au Sénégal est aussi celle sur laquelle la plupart des recherches se sont concentrées (Diop, 1990) ; (Bava, 2003). À côté de la Murîdiyya existe la Tijâniyya (la plus grande en nombre d'adeptes), la Quadiriyya et les Layène. 
2003. Là-bas c'était bien. Je gagnais bien ma vie. Seulement il n'y avait pas beaucoup de Sénégalais comme en France ou en Italie. J'avais loué un appartement avec deux Nigérians. Mais mes parents aussi bien au Sénégal qu'ici ne cessaient de me demander de revenir en France où se trouvent les autres membres de ma famille. »

Parmi les Sénégalais, les territoires autres que les pôles d'installation défrichés par les aînés sont qualifiés de "dem alla $b a$ », que l'on pourrait traduire par "brousse », et qui désigne des espaces non humanisés, un anti-écoumène. Ce phénomène de contrôle social pourrait être également observé sous d'autres formes parmi les Marocains ou dans d'autres types de migrations de travail, notamment ouvrières. Il montre que l'acte migratoire est inséré dans un dispositif social et familial qui contraint les acteurs à conserver des liens avec la communauté d'origine. Ce dispositif prémunit contre l'affaiblissement des liens sociaux entre les migrants et les personnes qui n'ont pas pu émigrer. Le lien avec le pays n'a donc rien de naturel, c'est une construction qui évolue et se transforme avec le temps, au cours du parcours migratoire. Comme le soulignent Deborah Brycesson et Ulla Vuorela (2002), les familles transnationales ne font qu'expérimenter de manière plus évidente le caractère construit de l'institution familiale en expérimentant la problématique de l'unité dans la dispersion géographique. L'une des principales conséquences est de garantir la rente économique que constituent les expatriés. On comprend alors d'autant mieux l'ampleur et la persistance du phénomène des transferts. Ces derniers ont atteint près de 4,8 milliards d'euros en 2006 vers les Maroc, soit l'équivalent de la moitié du déficit commercial. Quant aux envois des Sénégalais, ils sont estimés à 472 millions d'euros sur la même année.

Ce fonctionnement communautaire constitue, selon nous, directement ou indirectement, le soubassement de l'ensemble des relations transnationales. Ceci ne signifie pas que le transnationalisme est un phénomène de repli communautaire. En effet, l'ancienneté des migrations marocaines et sénégalaises en France est un facteur de diversification des relations transnationales. Aujourd'hui les mécanismes d'intégration, les nouvelles générations, les nouveaux profils socioprofessionnels, mais aussi l'évolution des sociétés d'accueil et d'origine, ont profondément marqué les pratiques qui sont de plus en plus ouvertes sur les groupes extracommunautaires.

\section{TRANSNATIONALISME SOCIOCULTUREL}

Les pratiques culturelles (alimentation, pratiques religieuses, engagements associatifs, goûts musicaux, pratiques festives etc.) sont directement affectées par les évolutions des groupes sociaux et sont donc traversées de tensions et de contradictions. Nous distinguerons ici les domaines religieux, folkloriques et associatifs pour mettre en évidence la façon dont ces pratiques se transforment grâce à l'expérience migratoire. Cette dernière a pour conséquence l'émergence de pratiques inédites au sein d'un espace social transnational, englobant davantage de variantes que la somme des composantes issues du pays d'origine et des différents pays de résidence. 


\section{La dimension du religieux : appartenance au groupe et autonomisation politique}

Les premiers commerçants sénégalais sont des Mourides. Le fonctionnement de cette confrérie s'est d'abord fondé sur une économie agricole de la culture arachidière. C'est pour faire face au déclin de cette ressource à partir des années 1960, que les Mourides ont investi le petit commerce urbain en Europe et aux États-Unis. De fait, le contrôle des talibés (disciples) par leur hiérarchie deviendra de plus en plus indirect et se fera à distance. En France, ce contrôle est réalisé grâce au réseau des maisons de Serigne Touba ${ }^{4}$. Les visites des Cheikhs dans ces maisons, permettent de réaffirmer les relations avec les talibés, notamment les obligations envers les Cheikhs.

La mise en place des maisons de Serigne Touba en France, Italie, États-Unis constitue une stratégie pour regrouper les disciples dans des endroits précis afin de mieux les contrôler et de poursuivre leur socialisation. Ces lieux repères sont de véritables points nodaux du transnationalisme religieux et identitaire mouride comme en atteste le discours de ce Cheikh dans la maison de Serigne Touba d'Aulnay Sous Bois :

"Les Mourides doivent être unis parce que la communauté mouride a besoin de talibés qui ont la force physique, de talibés qui ont de la richesse et de ceux qui ont le savoir-faire, la clairvoyance d'esprit et de bonnes idées. Tous ces talibés que j'ai cités là se complètent... Les talibés qui sont en France, en Italie, en Espagne et aux États-Unis doivent faire vivre les maisons de Serigne Touba en les fréquentant et en venant y apprendre ce qu' est être mouride, pour pouvoir diffuser le message de Serigne Touba. Aujourd' hui, c'est grâce à Serigne Mourtada que nous avons des maisons de Serigne Touba un peu partout en Europe et même au États-Unis. De toute façon Serigne Touba l'avait prédit en son temps... : "Ceux qui me persécutent (Il s'agissait de l'administration coloniale) ici, je viendrai leur parler et les convaincre jusque chez eux et ce sont même leurs descendants qui vous aideront à répandre ma parole et mes enseignements." Ce qui se vérifie absolument... Aujourd' hui on a beaucoup de toubabs convertis au mouridisme et qui nous aident à enseigner les enfants de mourides, que ce soit en France, au États-Unis, en Espagne, en Italie et aux États-Unis ».

4 Les maisons s'appellent Keur Serigne Touba en wolof. «Keur » signifie « maison »; « Serigne » désigne un guide spirituel et se traduit en français par «marabout ». Le nom des maisons se réfère à Cheikh Ahmadou Bamba (1850-1927), fondateur de la confrérie mouride au XIX ${ }^{\mathrm{e}}$ siècle, et dont le siège se trouve dans la ville sénégalaise de Touba.

5 Cheikh Mourtala Mbacké est l'un des fils de Cheikh Ahmadou Bamba qui est le marabout par excellence des disciples mourides en migration. Il faisait des allers-retours incessants entre le Sénégal et les pays d'installation des Mourides comme les États-Unis, la France, les autres pays de l'Union européenne et même l'Asie. À chacune de ses visites, est organisé un rassemblement par les talibés pour l'accueillir et recevoir ses bénédictions. C'est grâce à lui que les Mourides se sont organisés à travers les maisons de Serigne Touba. 
Ce discours rappelle les principes fondateurs et organisationnels de la confrérie. La soumission des disciples à l'égard de la hiérarchie maraboutique trouve son importance dans les sociétés d'accueil occidentales où les disciples sont confrontés à un univers de références potentiellement subversives. Quelques idées fortes peuvent y être décelées et notamment la nécessité d'une unité des disciples puisqu'ils se complètent selon leurs compétences. Les talibés se doivent de fréquenter régulièrement les maisons de Serigne Touba et de consacrer un travail intense, dans un esprit de sacrifice, à la confrérie. Nous retrouvons aussi, dans ce discours, le thème de la colonisation symbolique, le retour des Mourides qui viennent chez l'ancien colonisateur lui parler et convertir sa descendance. Le transnationalisme mouride comporte ainsi une part importante de renversement symbolique des rapports de domination.

Ces relations maîtres/disciples paternalistes sont toutefois aujourd'hui remises en question. Nous pouvons observer un certain détachement des jeunes pratiquants des consignes de leurs guides spirituels. L'éloignement géographique, en dépit des gardefous, présente aussi l'occasion de se distancier du contrôle social, et de réduire ses engagements financiers vis-à-vis du marabout. Cette prise de distance, minoritaire, concerne surtout la seconde génération de Sénégalais nés en France, et certains intellectuels en quête d'autonomie.

Chez les Marocains, le rapport au religieux est fondamentalement différent dans la mesure où le chef de d'État est également celui de la communauté des Musulmans. L'État exerce donc un contrôle étroit des activités religieuses, y compris au sein de la communauté marocaine à l'étranger. Ce contrôle s'est accru avec l'émergence d'une opposition islamiste. Cette nouvelle opposition a coïncidé avec l'affaissement de la gauche radicale marocaine après 25 ans de répression (voir ciaprès). À la fin des années 1980, le système de contrôle politique des Marocains de l'étranger a connu une profonde mutation. Le système précédent, fondé sur le réseau des «Amicales de commerçants et entrepreneurs », un réseau d'associations pilotées par le réseau consulaire et chargées d'encadrer la population ouvrière, est remplacé par la prise en main d'un réseau de mosquées et d'associations islamiques réparties en France. La création du Conseil Français du Culte Musulman en 2003 a placé sur le devant de la scène deux organisations marocaines ayant par ailleurs des relations transnationales de nature très différentes. La première est la Fédération Nationale des Musulmans de France (FNMF), dirigée par un proche des autorités marocaines. La seconde organisation est l'Union des Organisations Islamiques de France (UOIF), animée par des acteurs nés en France. Les relations transnationales développées par l'UOIF sont davantage orientées vers l'Égypte et le Moyen-Orient que vers le pays d'origine.

Le religieux est donc traité de façon radicalement différente parmi les Marocains et les Sénégalais. Chez ces derniers, la confrérie mouride a produit un dispositif de cadrage identitaire, spatial et spirituel, doublé par un dispositif économique transnational. Chez les premiers, le religieux est un champ de tensions politiques sur lequel s'exerce le contrôle de l'État marocain. 


\section{Le transnationalisme folklorique et intellectuel}

Le transnationalisme folklorique se manifeste sous différentes formes : création d'associations culturelles et allers-retours d'artistes et de musiciens dans le cadre de concerts et de festivals. Nous distinguons ici les activités culturelles faites par et pour des migrants dans le but d'animer la vie collective du groupe de leurs dérivés artistiques, commerciaux et militants qui sont apparus au fil des ans.

Les artistes, présents depuis les débuts de l'immigration, animent fêtes, mariages, événements religieux et festivals. Ils accompagnent la vie sociale transfrontalière des migrants. Le cas suivant illustre l'importance de cette sphère d'activité : M'Hamed est un chanteur de longue date, il dispose de son propre groupe au Maroc depuis qu'il a 17 ans. Il est restaurateur la semaine et chanteur le week-end. Il est sollicité principalement pour des mariages, mais aussi pour des soirées associatives ou des fêtes. La dimension transnationale de son activité apparaît, lorsque, en été, il retourne au Maroc pour réintégrer son groupe d'Agadir et où il arpente la région pour participer là encore aux mariages et aux fêtes locales. Aujourd'hui, M'Hamed dispose donc de deux groupes, l'un en région parisienne, l'autre à Agadir. Il a par ailleurs fait un disque produit par une maison d'Agadir. L'album s'est vendu au Maroc et a été également diffusé en France, en Espagne, en Belgique et aux Pays-Bas.

Le transnational est, par ailleurs, le champ d'expression d'artistes d'envergure internationale, en particulier sénégalais : le Super Étoile de Youssou Ndour donne chaque année un concert à Bercy, Baba Maal vient très régulièrement à Mantes-la-Jolie dans la communauté Haal Poulaar. Si la plupart des artistes sont installés au Sénégal et viennent dans les pays de l'espace Schengen lors d'événements culturels, il y a, à l'inverse, de plus en plus de petits artistes qui s'installent en France et retournent fréquemment au Sénégal, à l'exemple de Touré Kounda, Kiné Lam et Ablaye Mbaye.

À côté des artistes, diverses associations ont investi le champ culturel à des fins qui peuvent parfois émarger sur le politique. Parmi les associations populaires Amazigh $^{6}$, la majorité cherche à promouvoir la culture berbère, mais certaines ont entrepris une démarche politique de revendication identitaire, dans la foulée du mouvement berbériste initié en Algérie. Les organisations militantes sont regroupées au sein du congrès Mondial Amazigh, créé en 1995. Ce regroupement rassemble une centaine d'organisations du Maroc, d'Algérie, du Niger, du Mali et d'Europe, etc.

D'autres associations ont un arrière-plan intellectuel plus marqué. Il s'agit d'associations travaillant au rapprochement interculturel par l'organisation de manifestations autour de la culture et la vie marocaine, par exemple les associations Comara et Formidec dans la région Rhône-Alpes. Animées par des cadres et des universitaires, elles combinent leurs activités en France à des actions culturelles et de développement

6 Le terme Amazigh désigne les organisations berbéristes. Il est un substitut au terme « berbère » considéré comme dégradant et impropre à cause de son étymologie identique à celle de «barbare ». 
social au Maroc. Il faut aussi ranger dans cette catégorie des structures qui animent un ou des sites Internet à destination des Marocains de l'étranger. Ces sites sont très variés, de durée de vie et de portée diverses. Le plus important est Yabiladi, créé en 2001.

Le transnationalisme culturel inclut bien d'autres dimensions que des pratiques proprement culturelles. En effet, Il y a une forte imbrication entre le culturel, le politique et l'économique. Cette porosité se manifeste, par exemple, par l'émergence d'une classe d' « artiste-entrepreneur » à l'image de Youssou Ndour. Aujourd'hui ce domaine du transnational compte une grande variété de pratiques. À côté des activités motivées par un besoin nostalgique et communautaire, s'épanouissent des initiatives qui sont de véritables ponts interculturels. Le transnational est donc aussi la matrice d'un vivre-ensemble entre groupes ayant migré ou non/nationaux et non-nationaux.

\section{ENTREPRENEURS MIGRANTS ET ACTEURS DE DÉVELOP- PEMENT}

Par transnationalisme économique, il faut entendre aussi bien les investissements individuels effectués dans un cadre transnational, les investissements collectifs pour soutenir le développement local mais aussi les activités entrepreunariales s'étendant sur plusieurs États simultanément. Le transnationalisme économique est sous-tendu par une forte logique communautaire. Toutefois, nous verrons à travers les activités associatives de développement, que ce communautarisme fondateur peut être dépassé. Les pratiques de développement associent de plus en plus d'acteurs extérieurs : volontaires français, bailleurs de fonds, etc.

\section{La traversée de frontière : une ressource commerciale}

Les réseaux commerciaux transnationaux marocains ont fait l'objet de nombreux travaux depuis une vingtaine d'année (Tarrius, 2002 ; Battegay, 2003 ; Ma Mung et al., 1996). Certains groupes marocains comme les Soussis connaissent un savoir-faire commercial. Ils sont particulièrement présents dans le secteur de la vente au détail tant en France qu'au Maroc. Mais ils le sont également dans la confection, le transport ou les biens culturels (cassettes vidéo et audio...). Le système commercial soussi repose sur un agencement social communautaire.

Lors de nos enquêtes nous avons pu mener une série d'entretiens sur la place marchande de Asnières-Gennevilliers, dans le quartier de la place Voltaire. Il s'agit d'une plateforme commerciale historique, dans la mesure où elle fut l'une des premières de cette importance en France. Pourtant, très peu d'études lui ont été consacrées à ce jour (El Hariri, 2002 ; Aït Ouaziz, 1989). Nous avons dénombré dans la zone de la place Voltaire 72 boutiques tenues par des Marocains : épiceries, mobilier, téléboutiques, bazar, boucheries, boulangeries, etc. Le quartier fait, par ailleurs, office de gare routière marocaine à partir de laquelle les liaisons, par bus pour les personnes et mini-bus pour les biens, sont possibles en direction du Maroc et des autres pays 
d'accueil. Nous avons recensé 14 agences de voyage. L'origine de cette présence importante est liée à la venue d'un chef de tribu berbère, Moumoulhous, après l'indépendance du pays, en 1956. Grâce à sa fortune, il devient propriétaire d'un immeuble qu'il loue à des immigrés issus de la même tribu. Il fait ensuite l'acquisition d'une épicerie, puis de matériel de confection. Il achète le tissu auprès de commerçants juifs marocains du Sentier et fait confectionner des robes de style arabe (caftans, etc.). Moumoulhous aide les commerçants à s'installer et leur fournit l'achalandage en échange d'un contrat moral d'exclusivité en matière de fourniture. La plupart de ces commerçants sont d'anciens ouvriers qui ont ouvert une échoppe avec leurs économies et des prêts obtenus grâce à des proches (le système bancaire ne serait le recours que chez $20 \%$ d'entre eux (Ma Mung et al., 1990 : 63). Ce système s'est peu à peu généralisé avec l'émergence de nouveaux fournisseurs et la diversification de l'achalandage, mais ses structures sont restées identiques. La réputation, la parole donnée et le don fondent ce système plus sûrement que le contrat et le droit. Le recrutement de la main-d'œuvre se fait dans l'entourage familial élargi. La clientèle visée est essentiellement communautaire. Les produits sont en provenance ou à destination du Maroc, dans le but d'une consommation nostalgique ou encore pour des cadeaux à la famille. Cet encastrement du commerce et des rapports sociaux entretient une forte cohésion communautaire où le contrôle social est à la mesure des ressources auxquelles ses membres ont accès.

Le modèle commercial sénégalais, qui a le plus retenu l'attention des chercheurs, est celui de la confrérie Mouride, un réseau dont les centralités principales sont Touba au Sénégal (la capitale religieuse), New York et Paris et qui étend ses ramifications dans le monde entier (Diop, 1990 ; Riccio, 2000 ; Salzbrunn, 2007 ; Schmitz, 2003). Nous préférons ici nous attarder sur le commerce sédentaire sénégalais non mouride, qui est beaucoup moins connu, mais dont le déploiement transnational est tout aussi inventif. Nous prendrons le cas de Dramé, une entreprise commerciale sénégalaise du $18^{\mathrm{e}}$ arrondissement à Château Rouge, spécialisée d'abord dans la vente de cassettes, étendue ensuite au commerce de produits cosmétique. Dramé est gérée par

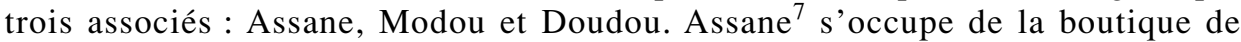
cosmétique, Modou de la boutique de cassettes et de CD tandis que Doudou fait des allers-retours entre le Sénégal et la France. Ce dernier a une formation de cameraman et filme des pièces de théâtre au Sénégal. Dramé a signé des contrats d'exclusivité avec des troupes de théâtre au Sénégal ; les prestations sont payées entre 500 à 650 euros. Dramé réalise le fractionnement des œuvres en plusieurs épisodes enregistrés sur des cassettes et des CD fabriqués en Belgique par une entreprise partenaire.

Dramé constitue donc un exemple de commerce qui repose sur une circulation transnationale active. À ce titre, il correspond à la définition que donnent Alejandro Portes et Louis Eduardo Guarnizo (2002) des entrepreneurs transnationaux, c'est-à-dire des migrants qui dirigent une entreprise dont le fonctionnement dépend de l'étranger (le pays d'origine dans la majorité des cas) où le commerçant est obligé de se rendre au moins deux fois par an dans le cadre de son activité économique. Néanmoins, une telle

7 Les noms ont été modifiés. 
définition demeure problématique et n'embrasse pas la totalité des cas rencontrés. Elle fait sortir de la catégorie d'entrepreneurs transnationaux par exemple les vendeurs présents sur les lieux touristiques qui s'approvisionnent chez les grossistes chinois du $3^{\mathrm{e}}$ arrondissement. Elle exclut également les détaillants présents sur les places marchandes, à l'instar de ceux de la place Voltaire. Or, même si ces commerçants n'ont pas par eux-mêmes d'activité circulatoire, ils constituent un chaînon essentiel des dispositifs marchands transnationaux. Cette définition des entrepreneurs transnationaux donc ne prend pas en compte ceux qui sont insérés dans un système organisationnel qui leur épargne la nécessité de circuler.

Entre la France et le Sénégal, des touristes « à la valise » jouent régulièrement le rôle d'intermédiaire entre Dakar et les différentes régions françaises où sont installés ces commerçants. Le dispositif marocain de Gennevilliers est lui doté d'un ensemble d'entreprises spécialisées dans le transport de marchandises qui effectuent des courses internationales à la demande. La circulation des acteurs n'est donc pas une condition nécessaire pour les définir en tant qu'acteur transnational.

\section{Les organisations de développement}

Les projets de développement peuvent être considérés comme une forme d'investissement collectif pour la communauté d'origine. Ce type de mobilisation collective, qu'il soit à caractère religieux, d'équipement public, social ou économique, est très répandu tant parmi les Sénégalais que chez les Marocains. Ces projets sont le fait de petits collectifs de villageois expatriés. Ces collectifs ont profondément évolué sous la pression de jeunes nés en France, de l'arrivée à l'âge de la retraite des premiers arrivants, mais aussi des effets de l'intégration qui génère de nouvelles préoccupations. Les exemples ici présentés illustrent cette évolution.

Les migrants de Ceedo au Sénégal ont constitué trois associations. La première, composée de personnes âgées, est orientée vers l'entretien des mosquées. La seconde est animée par les jeunes qui destinent leurs investissements aux activités sportives de Ceedo. La troisième est une association regroupant anciens et jeunes qui finance les projets de développement (forages, école, postes de santé, etc.). D'après le Secrétaire de cette dernière association :

"Nous n'avons pas pu nous entendre avec les vieux, c'est pour cela que nous avons jugé utile de créer ces trois structures. D'ailleurs l'association des vieux est informelle. Ils se rencontrent de temps à autre pour se cotiser et financer des travaux de la mosquée mais aussi pour les gamous ${ }^{8}$ au village. Ils n'ont même pas enregistré leur association à la préfecture et se sont regroupés par affinité ».

Ce type d'opposition générationnelle est également visible parmi les Marocains. Les jeunes introduisent une idée rénovée de l'aide au village, détachée des préoccupations coutumières et religieuses. Par ailleurs, ils mettent en œuvre des

8 Fête religieuse tijâne en honneur de l'anniversaire du Prophète. 
techniques associatives formalisées : enregistrement des statuts, tenue d'une comptabilité, demande de subvention, etc. Mais les dynamiques d'intégration et la diversité des générations ne sont pas les seuls facteurs d'évolution de ce type de pratiques. Une élite associative, composée d'anciens syndicalistes, de militants politiques, de réfugiés, a largement contribué à la structuration de ces groupes de porteurs de projets. Christophe Daum a relevé le rôle important de ce type d'acteur parmi les Maliens et les Sénégalais (Daum, 1998). Mais c'est surtout vrai pour les organisations marocaines en raison de l'activisme des réfugiés politiques (Lacroix, 2005). Ainsi, plusieurs ONG d'émigrés marocains ont été créées en France pour soutenir ces collectifs. La plus importante, « Migrations et Développement », est située à Marseille. Elle fut créée en 1987 par un ancien ouvrier de Péchiney qui fut également délégué syndical de la CFDT. Elle a aujourd'hui réalisé des projets dans plus de deux cents villages du Sud marocain grâce aux soutiens des États français et marocains, de l'Union européenne et de multiples ONG partenaires. L'association est également régulièrement consultée par les pouvoirs publics sur les dispositifs de co-développement. Le second exemple est celui d' "Immigration, Développement, Démocratie ». Cette structure fut créée en 1998 à Paris par des réfugiés politiques et militants marocains de gauche. Il s'agit pour ces acteurs de retrouver une action militante au Maroc. Leur premier projet fut la mise en place de bibliothèques en milieu rural.

\section{Les organisations professionnelles}

Les associations professionnelles ne sont pas à proprement parler des organisations économiques, cependant elles jouent un rôle de plus en plus important pour l'insertion des migrants qualifiés sur le marché du travail des pays d'accueil ou d'origine. Elles sont le fait d'étudiants et de personnels qualifiés dont le nombre a cru dans les années 1990. Le premier objectif de ces structures est celui de l'accueil, la circulation d'information, la formation, les aides à l'embauche, etc. Leur fonction dépasse, pour certaines, le cadre strictement professionnel. Ainsi l'Association des Informaticiens Marocains en France (AIMF) organise des sorties socio-culturelles «afin de souder les relations entre les membres et minimiser les effets du dépaysement ». Or ce type d'association déploie très souvent une partie de ses activités au Maroc. Cela consiste essentiellement en des projets de développement et des transferts de compétence attenants au domaine professionnel de l'organisation. Par exemple, l'AIMF expédie annuellement de l'équipement informatique pour des écoles marocaines. « Maroc Entrepreneur » fut créé en 1999 à l'instigation d'étudiants marocains en Grandes Écoles et Universités françaises (ESSEC, HEC, ESCP, EM-Lyon, X, Centrale, ... etc.). Ces étudiants ont pour préoccupation commune le retour au pays après l'obtention de leur diplôme. L'association se pose en intermédiaire entre ces demandeurs d'emplois et les entreprises basées au Maroc. Maroc Entrepreneur regroupe aujourd'hui le plus grand réseau de cadres supérieurs et d'étudiants marocains en France, soit près de 5000 membres : $60 \%$ sont des diplômés, $40 \%$ sont encore étudiants.

Ce même type d'organisations existe parmi les Sénégalais de France. Par exemple, l'Association des Travailleurs Sénégalais, existe depuis plusieurs décennies. 
Un de leurs objectifs est le transfert au Sénégal de droits sociaux (allocations, retraite etc.) acquis en France.

Au-delà des questions d'ordre strictement professionnelles, on constate que ces associations jouent un rôle analogue à celui des filières pour l'immigration ouvrière, à savoir une fonction de porte d'entrée sur la société française et de maintien de liens avec le pays d'origine.

\section{TRANSNATIONALISME POLITIQUE}

Les État sénégalais et marocain s'efforcent de mobiliser, d'encadrer et de contrôler leurs migrants respectifs pour gagner des élections, se maintenir au pouvoir ou contrôler leur image à l'extérieur. La forme des relations transnationales entre un État et ses migrants est largement conditionnée par la configuration du régime politique du pays d'origine et des pays de résidence en question. Il s'agit ici de souligner les convergences et les différences entre les deux cas d'étude.

\section{La société civile migrante Marocaine : de l'opposition à l'ouverture}

L'opposition marocaine à la monarchie constitutionnelle s'est constituée très tôt sur une base transnationale. En effet, jusqu'au milieu des années 1970, la migration marocaine est le fait non seulement de migrants économiques mais aussi de réfugiés ${ }^{9}$.

L'opposition à la monarchie constitutionnelle — imposée en 1961 —, et surtout la série d'attentats contre le monarque à la fin des années 1960, ont déclenché une vague de répression contre la gauche marocaine dont une partie a pris le chemin de l'exil. C'est Mehdi Ben Barka, le fondateur du parti socialiste marocain, qui va fédérer, dès 1962, l'ensemble des courants de la gauche exilée au sein de l'AMF (Association des Marocains de France). En 1982, les divisions internes vont déboucher sur une scission : l'aile radicale crée l'Association des Travailleurs Marocains de France (ATMF). Les activistes soutiennent le mouvement ouvrier dans l'industrie française alors en crise. À partir de la fin des années 1980, l'ATMF centre ses activités sur les droits sociaux et politiques des immigrés (soutien scolaire, lutte contre les discriminations, soutien au mouvement des sans-papiers et revendication du droit de vote aux élections locales). L'association ne s'adresse plus spécifiquement aux Marocains, mais à l'ensemble de la communauté immigrée d'origine maghrébine. En 2000 l'ATMF devient l'Association des Travailleurs Maghrébins de France. Elle s'inscrit sur un plan européen, en rupture avec le « maroco-centrisme » de l'AMF. Au début des années 1990, l'association siège à l'éphémère «Forum Européen des Migrants », un organe consultatif auprès de la Commission Européenne. Cependant, à la fin des années 1990, l'ATMF réinvestit la sphère politique marocaine avec la double question de la représentation parlementaire des Marocains de l'étranger et du développement. L'inves-

9 Les associations de réfugiés ont récemment fait l'objet d'une série de travaux (Lacroix, 2005 ; Bousetta, 2001 ; Daoud, 2002 ; Merizak, 2006). 
tissement dans le développement du Maroc est pour certains militants une voie pour soutenir l'émergence d'une société civile démocratique au Maroc. Cette idée est à l'origine de la création, déjà mentionnée dans cet article, d'Immigration, Développement, Démocratie. Le second terrain sur lequel les militants de l'ATMF effectuent leur « retour » est celui du vote des Marocains de l'étranger aux élections nationales marocaines. Les élections législatives de 2002 furent l'occasion d'une relance du débat sur l'exercice du vote à l'extérieur du territoire national. En novembre 2005, le roi Mohamed VI annonce le rétablissement d'une représentation parlementaire. Revenant sur l'annonce royale, le Ministère de l'Intérieur, en juin 2006, invoque des difficultés techniques pour la mise en place effective de la procédure de vote pour les élections suivantes, en septembre 2007. Dès l'été, un collectif d'organisations marocaines en Europe nommé «Al Monadara », dont l'ATMF fait partie, se met en place pour faire pression sur le gouvernement de Rabat. Ce réseau entretient le débat via Internet puis lors d'une rencontre organisée en Juillet 2006 à Rabat. En novembre de la même année, le roi enterre implicitement le projet de représentation parlementaire par l'annonce de la création du Haut Conseil de la Communauté des Marocains de l'Étranger, un organe consultatif chargé d'intervenir sur les affaires concernant les Marocains expatriés. Al Monadara est la manifestation de l'existence d'une société civile marocaine transnationale dynamique et capable d'interpeller les pouvoirs publics.

Aujourd'hui, la société civile migratoire marocaine n'est plus divisée entre tenants et opposants à la monarchie. De nouvelles associations sont actives dans la sphère politique. Nous avons déjà mentionné le tissu ancien des associations berbéristes, prises entre folklorisme et militantisme culturel. Leur nombre et leur visibilité ont récemment augmenté, pris par l'élan du mouvement berbériste au Maroc.

Les jeunes Marocains des deux rives sont également actifs au niveau transnational. Créée en 2003, l'Union des Jeunesses Euromaghrébines milite pour la constitution d'un Maghreb arabe démocratique. L'UJEM a mis en place un cycle de rencontres et journées d'études, dont le forum euro-maghrébin de la jeunesse. Le concept de « Maghreb Uni » de cette association fait écho à la « maghrébinité » portée par ses membres. Selon le président de l'association, il est une conséquence du regard de la société française. Les Marocains, Tunisiens et Algériens se retrouvent à un même niveau face à la désignation de «Maghrébin » que leur assigne la société. Leur activisme se construit à partir d'un vécu européen, vécu à travers lequel ils cherchent à prendre part au débat sur l'autre rive.

\section{Les réseaux transnationaux comme multiplicateurs de voix}

Pour les Sénégalais, les enjeux électoraux jouent un rôle déterminant dans les relations entre les migrants et la classe politique. Les relations particulières avec la France et l'importance numérique d'immigrés Sénégalais font de l'hexagone une base de campagne et un espace de compétition privilégiée entre partis politiques.

Avant de gagner les élections présidentielles en l'an 2000, Abdoulaye Wade a mené la campagne électorale pendant un an depuis son lieu de résidence en France. La stratégie de miser sur les réseaux transnationaux comme multiplicateurs de voix s'est 
traduite par une mobilisation importante de militants politiques sur le terrain francilien. La mobilisation individuelle de militants, et l'utilisation de différents médias sont les deux aspects qui constituent la base de la stratégie électorale wadienne, poursuivie pendant son mandat jusqu'aux campagnes électorales présidentielles et législatives de l'an 2007.

Pendant la compagne électorale de l'an 2000, Abdoulaye Wade a fait enregistrer une cassette destinée aux migrants sénégalais en France, aux États-Unis, en Italie, en Allemagne, et partout où réside une communauté importante de Sénégalais. Au cours de cette campagne, clôturée par sa première victoire, ainsi que pendant toute la durée de son mandat jusqu'à sa réélection en 2007, Wade a toujours misé sur l'importance des réseaux politiques transnationaux. Par l'intermédiaire de discours ou de vidéos enregistrés lors de meetings, il a créé une image d'ubiquité, tout en donnant aux migrants sénégalais de chaque pays de résidence l'impression de connaître leur situation particulière et de défendre leurs intérêts.

"C'est une excellente occasion pour moi que de m'adresser aux immigrés sénégalais et aux immigrés africains en général pour bien cadrer le problème de l'immigration et comprendre la politique que j'envisage de mener dans ce domaine-là... En tant que Libéral, je suis donc pour la liberté de déplacement des immigrés - africains comme des autres. Aujourd' hui, on compte à peu près 50000 immigrés sénégalais en France, 50000 immigrés aux États-Unis, peut-être 80000 en Italie. Donc, c'est vraiment mineur... Mais l'immigration africaine a certains aspects tout à fait particuliers... Après la guerre, la France avait besoin de main-d' euvre et a fait appel à des immigrés avec des salaires et des conditions très alléchantes. Puis,... les Français eux-mêmes ont eu besoins d'emplois, il y a eu un phénomène de rejet de l'immigration avec les discriminations... Et aujourd' hui... l'Europe va avoir besoin d'une main-d' 'euvre étrangère... Mais... pour l'Afrique,... il y a les aspects positifs. Il y a des immigrés qui envoient de l'argent en Afrique... Et ils nourrissent leurs familles ici. Et c'est autant de problèmes en moins pour le gouvernement qui est déjà sollicité par les problèmes de chômage... Mais... l'immigration présente des aspects négatifs pour nous. Notre pays se trouve privé d'une certaine main-d' œuvre... La plupart des immigrés viennent... des zones périphériques qui sont donc désertées et qui contentent de moins en moins de jeunes gens pour constituer... la main-d' 'euvre active et on y trouve que des enfants, des femmes et des vieillards, ce qui est très préjudiciable au développement de ces zones... Je considère que la première mesure à prendre est... de faire en sorte que les immigrés soient encouragés à revenir au Sénégal, périodiquement, et surtout qu'ils reviennent définitivement au bout d'un certain moment».

En 2000, Wade fit quelques promesses aux migrants, dont un contrôle plus juste par la douane sénégalaise de ceux qui rentrent au pays avec des « cadeaux ». Il s'engagea à négocier une extension des droits à l'allocation familiale des immigrés aux membres des familles restés au Sénégal. Il évoqua aussi la question du mauvais traitement des sans-papiers. En mentionnant son influence sur le gouvernement français, Wade tenta de gagner la confiance des immigrés qui espéraient que leur situation individuelle s'améliore. 
Au moment des élections de 2000, la situation économique difficile du Sénégal, les affaires de corruption et l'usure du pouvoir en place facilitèrent la création de la coalition du Sopi (le changement), fruit d'une alliance destinée à destituer l'ancien Président Abdou Diouf. En 2007, la conjoncture politique est nettement moins favorable car un certain nombre de promesses électorales n'ont pas été tenues, notamment celles attendues par les migrants. Grâce à Internet, à la radio FM satellite et aux téléphones portables, les informations circulent plus vite que jamais et les actions gouvernementales sont suivies de près par les Sénégalais résidant à l'étranger. Conscient de cette attention particulière, le gouvernement inscrit sa communication dans une logique transnationale : le Ministre des Sénégalais de l'Extérieur s'adresse régulièrement aux migrants, le député des Sénégalais de l'Extérieur en fait de même et le Président Wade tient compte des activités, notamment économiques et politiques, des émigrés, dans ses déclarations.

Récemment le Président a entrepris de nombreuses actions afin de ranimer le potentiel multiplicateur de voix des militants de l'extérieur. Une «Alliance Internationale d'Émigrés Sénégalais pour la Réélection du Président, Maître Abdoulaye Wade en 2007 » a été créé, avec des antennes particulièrement actives aux États-Unis et en Italie, où le «Mouvement des Sénégalais d'Italie pour la réélection d'Abdoulaye Wade (Mosiraw) » a vu le jour. En France, on assiste à des manifestations critiques et à celle de l'opposition composée en partie de membres du PS, auparavant au pouvoir. Ce constat se traduit par la multiplication de débats informels dans les lieux de rencontre (foyers de travailleurs, manifestations intellectuelles, culturelles et religieuses etc.). Le Forum Res publica organise régulièrement des débats sur la politique actuelle à Paris, le site Internet africlive initie des rencontres avec des hommes politiques sénégalais et les diffuse ensuite sur Internet, enfin les relations diplomatiques entre la France et le Sénégal sont régulièrement suivies et commentées.

Certaines positions critiques se traduisent par des manifestations, comme celle du 27 février 2007 devant le Consulat à Paris ${ }^{10}$. Une des revendications des électeurs Sénégalais de l'Extérieur est l'instauration du vote par Internet car le nombre réduit d'emplacements régionaux de bureaux de vote installés dans les pays de résidence décourage un grand nombre d'électeurs du fait des distances importantes à parcourir pour voter ${ }^{11}$. L'époque actuelle est marquée par une prise de conscience des inégalités

10 La Manifestation organisée par Res Publica le 27 juillet devant le Consulat du Sénégal à Paris, avait quatre revendications : « 1 . Le maintien des élections présidentielles comme prévu pour le 25 février 2007 ; 2 . La non réédition d'assassinat politique à l'instar de celui de $\mathrm{M}^{\mathrm{e}}$ Babacar Séye, ancien Vice-président du Conseil Constitutionnel et la consécration de l'impunité par la tristement célèbre loi "Ezzan"; 3. La démission du Ministre de l'Intérieur pour son incompétence et son incapacité à organiser des élections libres, transparentes et pacifiques ; 4. La disqualification des candidats à l'élection présidentielle qui seraient tentés de retirer leur candidature à des fins d'annulation de celles-ci sur le fondement de l'article 34 de la constitution. ».

11 L'Italie, plus souple dans l'autorisation d'installer des bureaux de vote, est devenu le premier pays d'immigration sénégalaise en Europe. Il a organisé une soixantaine de bureaux de vote pour les Sénégalais en 2007, alors que la France n'en comptait que 35 dans une douzaine de lieux. 
existant entre les habitants d'origine européenne et d'origine africaine en France. Le constat d'une discrimination dont souffrent particulièrement les Africains Subsahariens mène à l'émergence de pratiques politiques transnationales qui mêlent des références à l'histoire nationale et à l'histoire transnationale, notamment l'esclavage ${ }^{12}$.

Les militants politiques Sénégalais et Marocains se sont organisés sur le territoire français depuis les années 1960. Comme les Marocains sont exclus du vote, leurs stratégies d'influence politique sur leur pays d'origine prennent surtout forme au sein du tissu associatif. À l'inverse, tous les partis sénégalais disposent d'antennes en France dont l'influence lors des élections est importante. Après l'alternance politique de 2000, les voix critiques trouvent écho dans de nouvelles configurations de la société civile. Cette dernière recourt à des pratiques politiques très diversifiées, notamment du fait de l'utilisation d'anciens et de nouveaux modes de communication adaptée à la situation et au public concerné. La perception de la discrimination en France a une influence sur le discours et les pratiques politiques des immigrés Marocains et Sénégalais, et se répercute dans leurs associations. Des alliances maghrébines, puis d'autres groupements fondés sur le phénotype, voient le jour afin de défendre les intérêts des immigrés en France. Ils s'organisent à leur tour de manière transnationale en s'inspirant parfois du modèle américain.

\section{CONCLUSION}

L'étude des pratiques transnationales marocaines et sénégalaises nous a permis de mettre en évidence les invariants et les évolutions du phénomène transnational. Il est ancré dans la logique des filières migratoires destinées à préserver le lien avec le lieu d'origine (et donc la manne financière des transferts) en dépit de la distance. Cette cohésion communautaire a permis le développement de dispositifs commerciaux internationaux malgré l'absence de régulation légale. Le jeu de la parole donnée, de la réputation et le contrôle inhérent aux relations suffisent à garantir les transactions entre partenaires commerciaux. Dans le domaine culturel, un large éventail d'activités artistiques et associatives se sont développées sur le terreau de la nostalgie et du besoin de trouver des référents identitaires communs. Enfin, au niveau politique, le transnationalisme est un champ de réactivation de l'allégeance au pays d'origine, un comportement encouragé, voire suscité par les États marocain et sénégalais. Le champ transnational serait donc à première vue un défi à l'intégration dans la société d'accueil, notamment lorsque la discrimination vide de son contenu les promesses de cette intégration. Cependant, une telle analyse ne serait qu'une lecture bien éloignée de la complexité des rapports sociaux. L'évolution de l'immigration, l'importance croissante des nouvelles générations, l'arrivée à l'âge de la retraite des premiers arrivants, ont profondément modifié les pratiques. L'envoi d'argent aux familles n'est, par exemple, plus qu'un mode de transfert parmi d'autres, comme les initiatives de co-

12 Les résultats d'un récent Testing du Centre d'Analyse Stratégique auprès d'employeurs sont publiés sur le site www.strategie.gouv.fr et dans le numéro 5 de la revue Horizons Stratégiques (2007). 
développement. De même, l'acquisition de savoir-faire et le perfectionnement de la division du travail au sein des espaces transnationaux ont ouvert de nouveaux horizons économiques pour les migrants à même de s'affranchir à la fois des contraintes territoriales et des contraintes de la circulation. Le fonctionnement de ces espaces transnationaux est caractérisé par un fort ancrage local. Des productions culturelles inédites, composées de références plurielles, émergent et deviennent les vecteurs d'une communication renouvelée avec la société d'accueil et de départ. Enfin, et surtout, les activités politiques démontrent que le champ transnational dépasse les seules relations avec l'espace national d'origine. La transgression des logiques bilatérales mène à la création d'alliances inédites au sein de l'espace social transnational.

\section{Références bibliographiques}

AIT OUAZIZ R. Kia (1989) Les commerçants soussis dans l'agglomération parisienne : insertion spatiale et relation avec le pays d'origine, Thèse pour le doctorat, Université de Poitiers, Faculté de Lettre et Sciences Humaines, 314 p.

BATTEGAY Alain (2003) Les recompositions d'une centralité commerçante immigrée : la Place du Pont à Lyon, Revue Européenne des Migrations Internationales, 19 (2), pp. 9-22.

BAVA Sophie (2003) De la «baraka aux affaires»: ethos économico-religieux et transnationalité chez les migrants sénégalais mourides, Revue Européenne des Migrations Internationales, 19 (2), pp. 69-84.

BELBAH M., VEGLIA P. (2003) Pour une histoire des Marocains en France, Hommes et Migrations, (1242), pp. 18-31.

BERTONCELLO Brigitte, BREDELOUP Sylvie (1999) Le Marseille des marins africains, Revue Européenne des Migrations Internationales, 15 (3), pp. 177-197.

BOUSSETTA Hassan (2001) Immigration, post immigration politics and the political mobilization of ethnic minorities. A comparative case study of Moroccans in four European cities, Thèse pour le doctorat, Université catholique de Bruxelles, Département sciences sociales, $444 \mathrm{p}$.

BRYCESON Deborah, VUORELA Ulla (2002) Transnational families in the twenty-first century, in Deborah Bryceson et Ulla Vuorela Eds., The transnational family, Oxford, and New York, Berg, pp. 3-30.

DAOUD Zakia (2002) De l'immigration à la citoyenneté, itinéraire d'une association maghrébine en France : l'ATMF (1960-2003), Houilles, Mémoires de la Méditerranée, $238 \mathrm{p}$.

DAUM Christophe (1998) Les associations de Maliens en France : migrations, développement et citoyenneté, Paris, Editions Karthala, 253 p.

DIOP A. Moustapha (1990) L'émigration Mouride en Europe, Hommes et Migrations, 1132, pp. 21-24.

EL HARIRI Saâdia (2002) Espaces et pratiques des Marocaines en immigration : le cas des femmes installées à Gennevilliers, in Mohamed Charef Éds., Les migrations au féminin, Agadir, Éditions Sud-Contact, pp. 131-150.

FAIST Thomas (2000) The volume and dynamics of international migration and transnational social spaces, Oxford, Clarendon Press, $380 \mathrm{p}$.

FONDATION HASSAN II POUR LES MAROCAINS RÉSIDANT À L'ÉTRANGER (2003), Marocains de l'extérieur, Rabat, Organisation Internationale pour les Migrations, 397 p. 
GLICK SCHILLER Nina, BASCH Linda, SZANTON BLANC Cristina (1992) Towards a transnational perspective on migration: race, class, ethnicity and nationalism reconsidered, New York, New York Academy of Sciences, 258 p.

GLICK SCHILLER Nina, CAGLAR Ayse (2006) Towards a theory of locality, in migration studies: migrant incorporation and city scale, Poitiers, Colloque 1985-2005.

GLICK SCHILLER Nina, FOURON Georges-Eugène (2001) Long distance nationalism and the search for home, Durham/London, Duke University Press, 326 p.

KAUFMANN Jean-Claude (2004) L'invention de soi. Une théorie de l'identité, Paris, Armand Colin, $351 \mathrm{p}$.

LACROIX Thomas (2005) Les réseaux marocains du développement : géographie du transnational et politique du territorial, Paris, Presses de Sciences Po., 257 p.

MA MUNG Emmanuel, SIMON Gildas, GUILLON Michelle, BOUBAKRI Hassan, AIT OUAZIZ R. Kia (1990) Commerçants maghrébins et asiatiques en France agglomération parisienne et villes de l'Est, Paris, Masson, $129 \mathrm{p}$.

MERIZAK Moustapha (2006) Immigration, militantisme politique et mouvement associatif des marocains en France : des origines aux évolutions, Paris, Université Paris VIII, Département de sociologie, Thèse doctorat, $388 \mathrm{p}$.

PORTES Alejandro, HALLER William, GUARNIZO Luis Eduardo (2002) Les entrepreneurs transnationaux : une forme alternative d'adaptation économique des immigrés, in Michel Péraldi Éds., La fin des norias? Réseaux migrants dans les économies marchandes en Méditerranée, Paris, Maisonneuve \& Larose, pp. 51-89.

PRIES Ludger (1996) Transnationale soziale Räume. Theoretisch-empirische skizze am Beispiel der Arbeitswanderungen Mexico-USA, Zeitschrift für Soziologie 25 (6), pp. 456-472.

RAY Joanny (1938) Les Marocains en France, Paris, Librairie du recueil, Sirey, 406 p.

RICCIO Bruno (2000) Spazi transnazionali: esperienze senegalesi, afriche e orienti (3/4), pp. 17-25.

SALZBRUNN Monika (1996a) Organisations paysannes, confréries islamiques et structures étatiques dans le processus de transformation au Sénégal : deux études de village au SineSaloum, La transmission du savoir dans le monde musulman périphérique (16) : pp. 17-29.

SALZBRUNN Monika (2002) Hybridization of religion and political practises amongst West African muslim migrants in Paris, in Deborah Bryceson, Ulla Vuorela Eds., The transnational family New european frontiers and global networks, Oxford, Berg, pp. 217-229.

SALZBRUNN Monika (2007) Localising transnationalism: researching political and cultural events in a context of migration. Transnationalism and development: towards a North-South perspective, Centre for interdisciplinary Research, Bielefeld, COMCAD.

SCHMITZ Jean (2003) Du djihad à la migration internationale. La diaspora des républiques musulmanes de la Vallée du Sénégal, Thèse pour le doctorat, Université Paris V.

TARRIUS Alain (2002) La mondialisation par le bas: les nouveaux nomades de l'économie souterraine, Paris, Balland, 168 p. 


\title{
Marocains et Sénégalais de France : permanences et évolution des relations transnationales
}

\author{
Thomas LACROIX, Leyla SALL et Monika SALZBRUNN
}

Cet article présente les pratiques transnationales marocaines et sénégalaises dans les sphères politiques, économiques et socioculturelles. En dépit du parallélisme historique des migrations marocaines et sénégalaises, l'étude souligne les différences de contenu qui distinguent les deux champs transnationaux. Par exemple, la confrérie sénégalaise mouride a produit un double dispositif transnational spirituel et économique. Parmi les Marocains, le religieux est un champ de tensions politiques sur lequel s'exerce le contrôle de l'État marocain.

Mais plus qu'un simple catalogue, les auteurs mettent à jour les invariants et les voies d'évolution du transnationalisme. L'ancienneté des migrations marocaines et sénégalaises en France est un facteur de diversification des relations transnationales. Les mécanismes d'intégration, les nouvelles générations, les nouveaux profils socioprofessionnels, mais aussi l'évolution des sociétés d'accueil et d'origine, ont profondément marqué les pratiques qui sont de nature de plus en plus ouvertes sur les groupes extracommunautaires.

\section{Moroccans and Senegalese of France : Permanence and Evolution of transnational Relations}

\author{
Thomas LACROIX, Leyla SALL and Monika SALZBRUNN
}

This article presents the Moroccan and Senegalese transnational practices in the political, economic and socio-cultural spheres. In spite of the historical parallelism of Moroccan and Senegalese, the study highlights the content differences which distinguish the two transnational fields. For instance, the Senegalese Murid brotherhood relies on their spiritual sway faithful to build up economic transnational networks. Amongst Moroccans, religion is a field of political tensions on which the Moroccan state strive to keep a firm hold.

But, more than a mere enumeration, the authors shed light on invariants and paths of change of transnationalism. The age of Moroccan and Senegalese immigration to France is a factor of diversification of transnational activities. Mechanisms of integration, new generations, new occupational profiles, but also evolution of host and receiving societies, have deeply transformed practices which are increasingly opened outside ethnic communities. 


\title{
Marroquíes y Senegaleses de Francia : permanencias y evolución de las relaciones transnacionales
}

\author{
Thomas LACROIX, Leyla SALL y Monika SALZBRUNN
}

Este artículo presenta las prácticas transnacionales marroquíes y senegalesas en las esferas políticas, económicas y socioculturales. A pesar del paralelismo histórico de las migraciones marroquíes y senegalesas, este estudio pone de relieve las diferencias de fondo que distinguen los dos campos transnacionales. Por ejemplo, la cofradía senegalesa mouride produjo un doble dispositivo transnacional, espiritual y económico. En el caso de los marroquíes, lo religioso está atravesado por tensiones políticas controladas por el Estado marroquí.

No se trata aquí de catalogar estas prácticas, sino poner a la luz los invariantes y las evoluciones del transnacionalismo / los procesos de transformación del transnacionalismo. La antigüedad de las migraciones marroquíes y senegalesas en Francia es un factor que contribuye a la diversificación de las relaciones transnacionales. Los mecanismos de integración, las nuevas generaciones, los nuevos perfiles socio profesionales, pero también la evolución de las sociedades de llegada y de origen, han profundamente marcado unas prácticas que resultan cada vez más abiertas sobre los grupos extracomunitarios. 


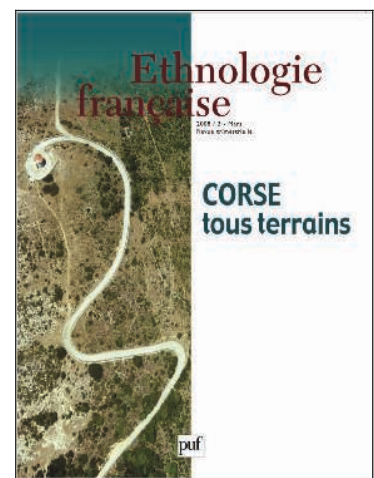

CORSE. TOUS TERRAINS

INTRODUCTION

Responsable scientifique

Charlie Galibert

L'ethnologie de la Corse : pistes et hors piste

\section{Le contexte socio-politique}

Elena Filippova

La Corse : une voix à part dans le concert français ?

Anne Meistersheim

$D u$ « riacquistu »au désenchantement. Une société en quête de repères

Wanda Dressler

La modernisation de l'île. Entre structures claniques et mouvements nationalistes

Marco Cini

Corse - Italie : proximité et fractures

[traduit de l'italien par Bernard Biancarelli]

André Fazi

Régions insulaires de Méditerranée occidentale : l'impossible autonomie?

\section{La recherche}

Max Caisson

Une denrée rare : l'ethnologie de la Corse

Philippe Pesteil

L'ethnologie au risque de la tutelle. Une discipline sous le couvert de la société civile

\section{CORSE \\ tous terrains}

Revue Ethnologie française $\mathrm{n}^{\circ} 2008 / 3$

Juillet-septembre 2008

\section{Responsable scientifique du numéro : Charlie Galibert}

Geneviève Michon et Jean Sorba

I Tré Valli. Passer par-dessus les montagnes

Eugène F.-X. Gherardi

La Vierge, les lycéens, la Corse. La "fête nationale » du 8 décembre

Bernard Biancarelli et Christine Bonardi

De quelques monstres anthropologiques insulaires Jean-Marie Arrighi

Langue corse : situation et débats

Alexandra Jaffe

Parlers et idéologies langagières

\section{Les institutions}

Jean Marc Olivesi

Musée de la Corse. Musée régional d'anthropologie

Dominique Verdoni

Inventaire et valorisation des patrimoines socioculturels

Un programme pour l'Université de Corse

\author{
VARIA : Expériences \\ Jean-Yves Bory \\ La polémique sur l'expérimentation animale. Le cas d'un \\ laboratoire de la sécurité routière \\ Maud Saint- Lary \\ Des vignerons franciliens en quête d'authenticité
}

COMPTES RENDUS

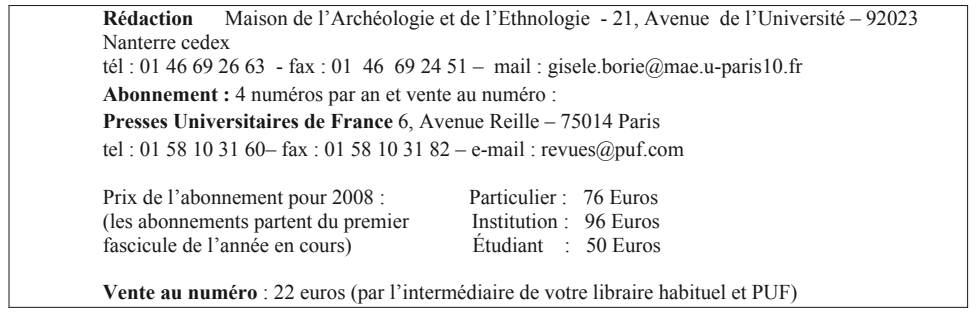

